\title{
A Compact Multipath Mitigating Ground Plane for Multiband GNSS Antennas
}

\author{
M. Maqsood, S. Gao, Member, IEEE, T. W. C. Brown, Member, IEEE, M. Unwin, R. de vos Van \\ Steenwijk and J. D. Xu
}

\begin{abstract}
This paper presents the design of a novel multipath mitigating ground plane for Global Navigation Satellite System (GNSS) antennae. Firstly, the concept of a compact low multipath cross plate reflector ground plane (CPRGP) is presented. In comparison to the choke ring and Electromagnetic Band Gap (EBG) ground planes, the proposed CPRGP has compact size, low mass, wide operational bandwidth and simple configuration. The proposed CPRGP is then integrated with a circularly polarized dual-band GNSS antenna in order to assess the multipath mitigating performance over two frequency bands. Measurement results of the proposed CPRGP with GNSS antenna achieves a front to back ratio (FBR) over $25 \mathrm{~dB}$ at L1 $(1.575 \mathrm{GHz})$ and $\mathrm{L} 2(1.227 \mathrm{GHz})$ bands and maximum backward cross polarization levels below $-23 \mathrm{~dB}$ at both bands. Antenna phase centre variation remains less than $2 \mathrm{~mm}$ across both $L 1$ and L2 bands. Furthermore, the performance comparison of the proposed CPRGP with the commercially available pinwheel antenna and the shallow corrugated ground plane is presented, showing the advantages of CPRGP for high precision GNSS applications.
\end{abstract}

Index Terms-Corrugated surfaces, global positioning system (GPS), multi frequency antennas, multipath interference

\section{INTRODUCTION}

$\mathrm{M}$ ULTIPATH error has remained a limiting factor in the achievement of centimeter level accuracy which is required for modern day GNSS applications. Narrow correlator spacing ( 0.1 chips) can suppress multipath delays greater than 1 chip which corresponds to 29.3 meters for 10.23 $\mathrm{MHz}$ chip clock [1]. However this is still not sufficient to achieve centimeter level accuracy and the need to suppress much shorter multipath signals has to be fulfilled with appropriate antenna design.

Multipath interference is the result of line of sight (LOS) signal replicas coming towards the antenna after reflection and diffraction from the surrounding environment and the nearby objects located at various azimuth angles. These signals are

Manuscript accepted for publication January 18, 2013.

M. Maqsood and T. W. C. Brown are with University of Surrey, UK (email: m.maqsood@surrey.ac.uk).

S. Gao was with Surrey Space Centre and is now at the University of Kent (e-mail: s.gao@kent.ac.uk)

M. Unwin and R. de vos van Steenwijk are with Surrey Satellite Technology Ltd., UK (e-mail: m.unwin@sstl.co.uk).

J. D. Xu is with Northwestern Polytechnical University, P.R. China (email: jdxu@nwpu.edu.cn). usually attenuated and depolarized in comparison to the original LOS signal and arrive at the antenna from random directions. In particular, the GNSS antenna is vulnerable to the low angle multipath interference signals as the co-polar signals of the antenna are usually weak in these regions [2].

The low angle multipath signals can be categorized into two types; signals coming after diffraction and signals coming after reflection. The diffracted signals are attenuated but may maintain their right hand circular polarization (RHCP) state. The direction of arrival of these signals is near the horizon and thus the antenna radiation pattern needs to have very low copolar power in order to reject them. The reflected signals on the other hand come from below the horizon in a left hand circular polarization (LHCP) state. These signals can be rejected if the antenna has very good cross polarization performance below the horizon.

Shaping the antenna radiation pattern is a powerful technique for suppressing multipath signals passively. Integrating an external ground plane with a circularly polarized antenna can help in tuning the antenna radiation pattern to achieve low co-polar gain beyond the required antenna beamwidth. The ground plane integration also improves the antenna cross polarization in the side and back lobes and thus can ensure a significant reduction in the multipath interference. However, it should be kept in mind that external ground plane integration will increase the overall mass and size of the antenna and may conflict with the design requirements. For GNSS antennas onboard small satellites or other small platforms, it is necessary to reduce the size, volume and mass of multipath mitigating GNSS antennas.

The choke ring ground plane is the most common ground plane used to shape the antenna pattern in order to mitigate multipath interference. It belongs to the family of electromagnetic soft surfaces which are used to suppress surface wave propagation within the antenna structure [3]. Although choke ring ground planes can effectively suppress surface wave propagation they are usually bulky and difficult to accommodate as they require $\lambda / 4$ deep corrugations with at least 3 to 4 concentric rings to achieve the desired performance.

In order to overcome the accommodation problem, a non cutoff corrugated ground plane with shallow corrugation depth is presented in [4] where surface waves are allowed to propagate but are tuned to cancel out with the LOS signals as 
they reach the edge of the ground plane. This technique reduces the ground plane thickness by $34 \%$ but still requires a large number of corrugated rings in order to demonstrate the concept.

Another method of reducing the height of choke ring ground plane is suggested in [5] where the choke ring cavities can be filled with high permittivity material to produce a dielectric loading effect. The design achieves a $68 \%$ reduction in the ground plane thickness and does not require a large number of rings. However, the cost of the antenna increases due to the requirement for high permittivity material.

In contrast to the corrugated ground plane, a low profile technique for surface wave suppression is the use of EBG substrate; a periodic structure creating a high impedance region around the antenna and blocking surface wave propagation. Although the EBG substrate is low profile it requires high periodicity and the overall antenna size needs to be very large in order to achieve good performance which leads to a large volume [6][7]. Due to the limited accommodation available onboard small platforms like small satellites, these EBG structures do not prove to be attractive multipath mitigating GNSS antennas.

This paper presents a novel design of a compact-size broadband external ground plane for multipath mitigation in GNSS antennas. The cross plate reflector ground plane (CPRGP) is capable of improving antenna co-polar and crosspolar performance about and below the horizon. The ground plane is simple, easy to manufacture and increases the overall antenna size by only $40 \mathrm{~mm}$. In order to demonstrate the ground plane performance, the CPRGP is integrated with a dual-band step-shorted annular ring (S-SAR) antenna.

Section II of the paper briefly presents the S-SAR antenna design and results, section III introduces the novel ground plane (CPRGP) and its integration with S-SAR antenna. CPRGP prototype manufacturing and measurement is presented in section IV while section $\mathrm{V}$ presents the comparative analysis of the proposed CPRGP against a commercial pinwheel antenna and non cutoff corrugated ground plane. Section VI concludes the paper.

\section{DUAL-BAND STEP-SHORTED ANNULAR RING (S-SAR) ANTENNA}

\section{A. Antenna configuration}

The dual band S-SAR antenna consists of two stacked circular ring elements with top and bottom rings resonating at L1 (1.575 GHz) and L2 (1.227 GHz) bands respectively. External and internal radii of both rings have been evaluated using the design equations 1 and 2 that implement reduced surface wave (RSW) boundary condition presented in [2][8-9]. A RSW microstrip antenna produces only a small amount of surface waves and its design relies on the principle that a circular patch modeled as a magnetic current ring (using cavity model) will not excite a $\mathrm{TM}_{0}$ surface wave as long as the radius ' $a$ ' of the circular patch is given by equation 1 .

$$
J_{1}^{\prime}\left(\beta_{\mathrm{TM}_{0}} a\right)=0
$$

which yields

$$
\beta_{\mathrm{TM}_{0}} a=x_{1 n}^{\prime}=1.8412,5.3314, \ldots
$$

where $a$ is the radius of patch [8].

In order to make a RSW antenna resonant at the same frequency at which the surface-wave mode is not excited, a circular short-circuit boundary condition concentric to the radiating edge must be implemented forming a shorted annular ring antenna [8]. The radius of the short circuit boundary can be evaluated using the transcendental equation

$$
\frac{J_{1}\left(k_{1} a\right)}{Y_{1}\left(k_{1} a\right)}=\frac{J_{1}^{\prime}\left(k_{1} b\right)}{Y_{1}^{\prime}\left(k_{1} b\right)}
$$

The above equation can be solved iteratively for the appropriate value of $b<a$. Four parameters namely $a_{L 1}, b_{L 1}$, $a_{L 2}$ and $b_{L 2}$ are evaluated for the two shorted annular ring patches. The optimized values of $a_{L 1}$ and $b_{L 1}$ are $52.8 \mathrm{~mm}$ and $23.8 \mathrm{~mm}$ while $a_{L 2}$ and $b_{L 2}$ are $54 \mathrm{~mm}$ and $15.2 \mathrm{~mm}$.
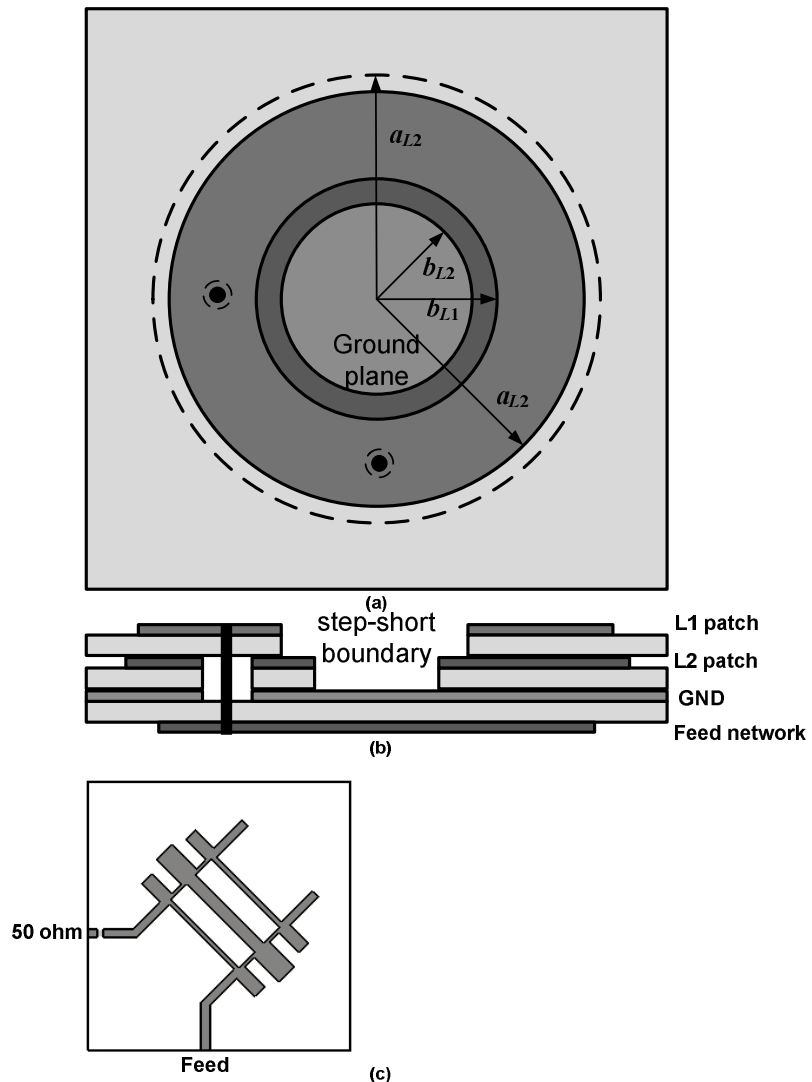

Fig. 1. (a) Top, (b) side and (c) bottom view of circularly polarized dual band S-SAR antenna with integrated feed network

Both stacked patch elements share a common ground plane that lies beneath the bottom ring with a step-shorted wall connecting inner boundaries of the two rings to the ground plane. A compact-size broadband microstrip hybrid coupler, as shown in Fig. 1(c), is designed and directly integrated with the antenna to achieve circular polarization. The feed network employs a cascaded hybrid coupler. A technique of lumped 
distributed transformation [10] is applied here to shorten the quarter wavelength sections of the hybrid.

Duroid $5880\left(\varepsilon_{\mathrm{r}}=2.2, \mathrm{~h}=1.575 \mathrm{~mm}\right)$ is used for the fabrication of the annular ring elements while FR4 $\left(\varepsilon_{\mathrm{r}}=4.55, \mathrm{~h}\right.$ $=1.6 \mathrm{~mm}$ ) is used for fabricating the feed network. The feed network is connected to the top antenna (L1 band) using vias which pass through clearance holes in the ground plane and the bottom patch. The bottom patch (L2 band) is electromagnetically coupled.

The S-SAR antenna structure presented in Fig. 1 was designed and simulated using the transient solver available in the 3D electromagnetic software Computer Simulation Technology (CST) [11]. The overall antenna dimensions are $150 \times 150 \mathrm{~mm}^{2}$.

\section{B. Antenna Results}

Simulation results from the software are compared with the measurement results for $s_{11}$ response and are presented in Fig. 2. A slight mismatch in the simulation and measurement results was expected as the annular rings were cutout using cone cutters of the nearest possible radii and the shorted rings were hand soldered leaving some mismatch in antenna dimensions but despite any errors in the fabrication, the antenna return loss is above $10 \mathrm{~dB}$ at both frequency bands for greater than $20 \mathrm{MHz}$ bandwidth. Fig. 3 and Fig. 4 present the comparison of normalized simulated and measured results of the dual band S-SAR antenna. The discrepancy in the cross polarization performance at the L1 band can be attributed to the human errors in the antenna manufacturing process.

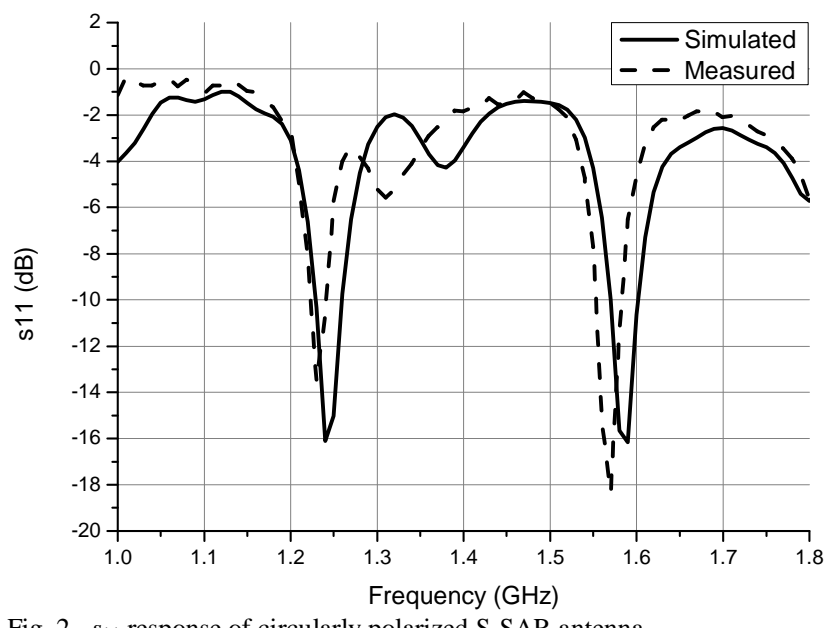

Fig. 2. $s_{11}$ response of circularly polarized S-SAR antenna

Table I shows the measured antenna gain against frequency bandwidth and it can be seen that the S-SAR antenna achieves a gain of more than $5 \mathrm{dBic}$ at both bands. Although the antenna performance has been optimized, the measurement results presented in Fig. 3, Fig. 4 and Table II suggest further improvement to mitigate multipath interference.

Improvement is required in the axial ratio beamwidth, the RHCP/LHCP ratio at the horizon and backward cross polarization performance for high end multipath mitigation.

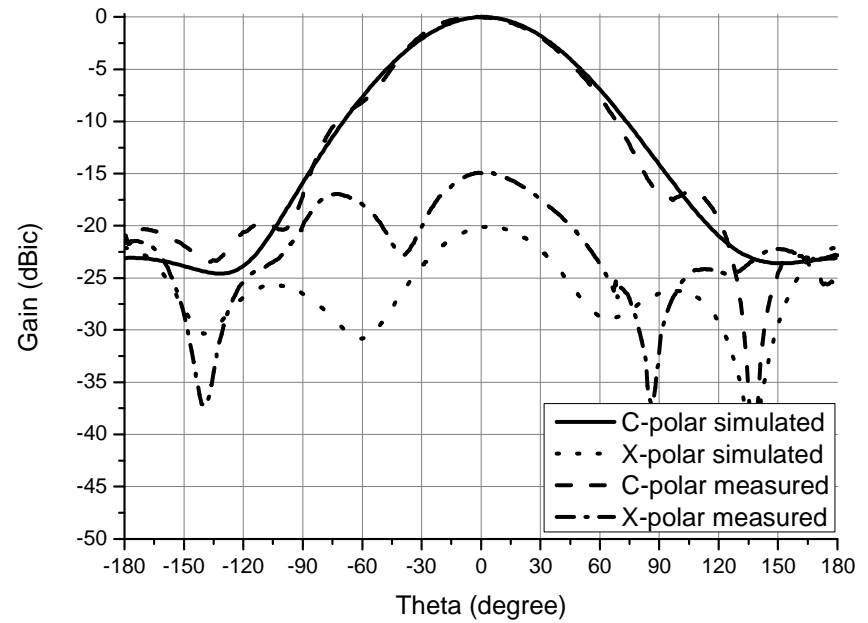

Fig. 3. Comparison of normalized simulated and measured radiation pattern of the S-SAR antenna at $1.227 \mathrm{GHz}$

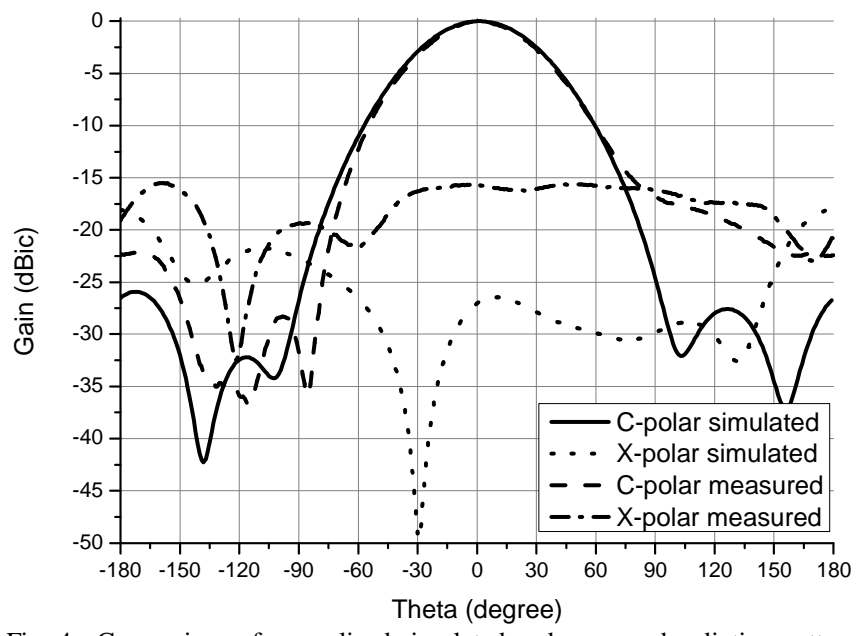

Fig. 4. Comparison of normalized simulated and measured radiation pattern of the S-SAR antenna at $1.575 \mathrm{GHz}$

\section{COMPACT MULTIPATH MITIGATING GROUND PLANE - CROSS PLATE REFLECTOR GROUND PLANE (CPRGP)}

Generally a good multipath mitigating GNSS antenna would require backward radiation of $\leq-10 \mathrm{dBic}$, a front to back ratio greater than $25 \mathrm{~dB}$, and the polarization isolation to be $\geq 15$ $\mathrm{dB}$ for the entire upper hemisphere [4]. Moreover, the pattern roll off from zenith to horizon should be between 8 and $14 \mathrm{~dB}$ while phase center variation should be less than $2 \mathrm{~mm}$ [12].

In order to meet the multipath mitigating criterion, the SSAR antenna is integrated with a novel multipath mitigating ground plane; the cross plate reflector ground plane (CPRGP). The proposed ground plane is simple, compact and low cost. The ground plane design is briefly presented in [13] but the following will describe in details; the design concept, manufacturing, and integration of the CPRGP with the S-SAR antenna.

\section{A. Design Concept}

The design of the novel cross plate reflector ground plane can be easily explained by using basic electromagnetic theory. Fig. 5 presents two types of oblique plane TEM waves; $\mathrm{TE}_{\mathrm{z}}$ 
(TE to $\mathrm{z}$ ) and $\mathrm{TM}_{\mathrm{z}}(\mathrm{TM}$ to $\mathrm{z}$ ) with the propagation vector in positive $\mathrm{x}$-direction. It is well known from electromagnetic theory that a flat perfect electric conductor (PEC) sheet will cancel out any type of plane waves on its surface if the E-field vector of the propagating wave is parallel to the PEC surface. Therefore, a flat metal sheet in $x-y$ direction will block the propagation of $\mathrm{TE}_{\mathrm{z}}$ waves but can not cancel $\mathrm{TM}_{\mathrm{z}}$ waves as the E-field vector is perpendicular to x-y plane. On the other hand, a ground plane geometry where the PEC sheets are arranged in a way that the E-field vectors of both types of plane waves are parallel to the PEC surface can effectively block their propagation.
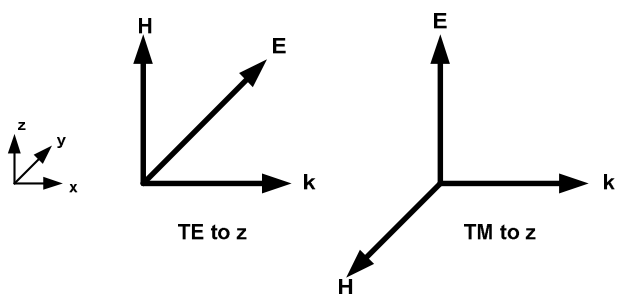

Fig. 5. Plane waves vector orientation in the Cartesian coordinate system

The above concept is further clarified in Fig. 6 where a portion of the CPRGP structure is shown. The thickness of the top surface and the flat metal bedding provides $E_{t}=0$ condition for the $\mathrm{TE}_{\mathrm{z}}$ waves while the vertical plates provide a similar condition for the $\mathrm{TM}_{\mathrm{z}}$ waves. Therefore, it can be concluded that the CPRGP provides $\mathrm{E}_{\mathrm{t}}=0$ for both types of TEM waves $\left(\mathrm{TE}_{\mathrm{z}}\right.$ and $\left.\mathrm{TM}_{\mathrm{z}}\right)$ and can cancel out both of them if the propagation vector is parallel to the ground plane surface.

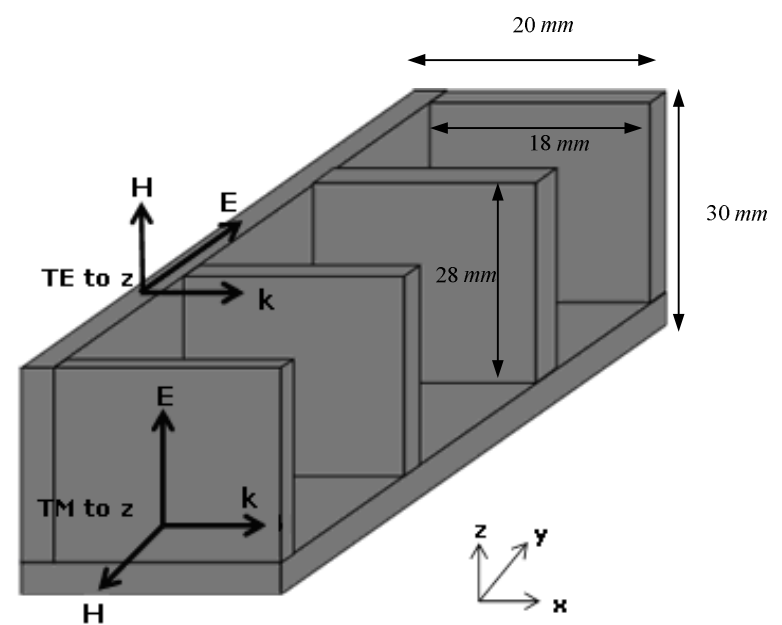

Fig. 6. Vector representation of $\mathrm{TE}_{\mathrm{z}}$ and $\mathrm{TM}_{\mathrm{z}}$ waves on the cross reflector ground plane surface

Simulation results of the CPRGP integrated with S-SAR antenna have shown that the ground plane structure is capable of achieving high end multipath mitigating performance while having a smaller volume. Performance improvement across both the L1 and L2 bands, shown in Fig. 7 and Fig. 8, signifies the broadband nature of the proposed ground plane structure. Antenna simulation results show that CPRGP integration improves the front to back ratio (FBR) by $7 \mathrm{~dB}$ at both bands.
The antenna cross-polarization below the horizon stays less than $-25 \mathrm{~dB}$ for most of the $3 \mathrm{~dB}$ beamwidth with the maximum value to be $-21 \mathrm{~dB}$ at $\mathrm{L} 2$ band.

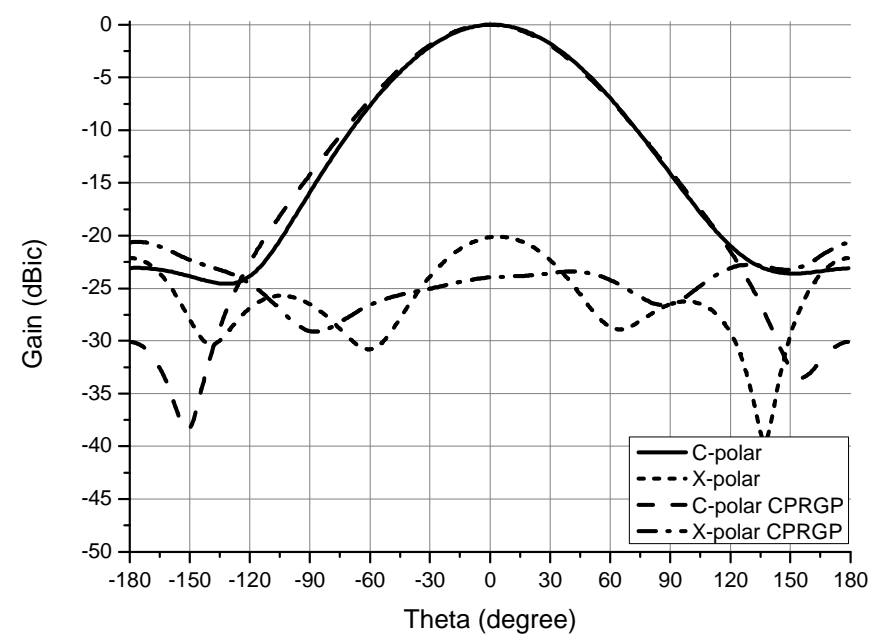

Fig. 7. Simulated radiation pattern (normalized) of the S-SAR antenna with and without CPRGP integration at $1.227 \mathrm{GHz}$

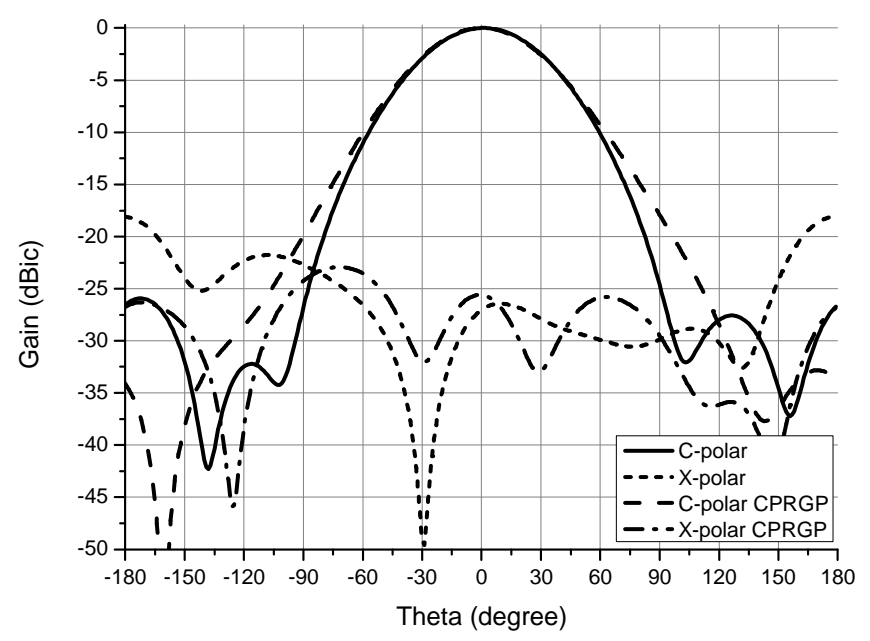

Fig. 8. Simulated radiation pattern (normalized) of the S-SAR antenna with and without CPRGP integration at $1.575 \mathrm{GHz}$

\section{CPRGP PROTOTYPE MANUFACTURING AND MEASUREMENT RESULTS}

In order to validate the performance improvement shown in Fig. 6 and Fig. 7 prototypes of dual band S-SAR antenna and cross reflector ground plane were manufactured and integrated. The CPRGP was manufactured using $2 \mathrm{~mm}$ thick aluminum plate. The simple geometry of the ground plane allowed for easy manufacture. Instead of milling the ground plane out of a solid block, small pieces and strips of an aluminum sheet were cut and glued together to a base plate. Holes were drilled in order to provide clearance for feed pins. The antenna and ground plane were integrated together by the nylon screws. Fig. 9 shows the manufactured prototype antenna integrated with the CPRGP. The integrated antenna was measured for return loss and radiation pattern. The 
antenna return loss curve is presented in Fig. 10 while normalized gain patterns are presented in Fig. 11 and Fig. 12. Table I presents the antenna gain across the coverage bandwidth showing a $5.8 \mathrm{dBic}$ gain at $1.227 \mathrm{GHz}$ and $7.7 \mathrm{dBic}$ gain at $1.575 \mathrm{GHz}$. The integrated multipath mitigating antenna covers $20 \mathrm{MHz}$ bandwidth at both L1 and L2 bands.

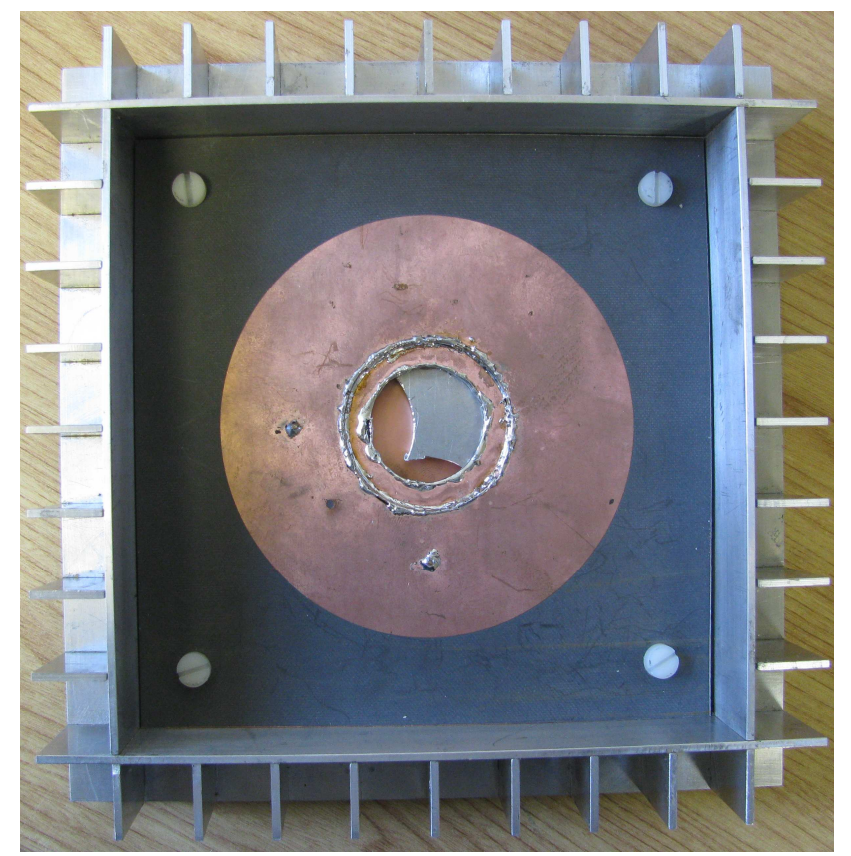

Fig. 9. Antenna prototype integrated with cross reflector ground plane.

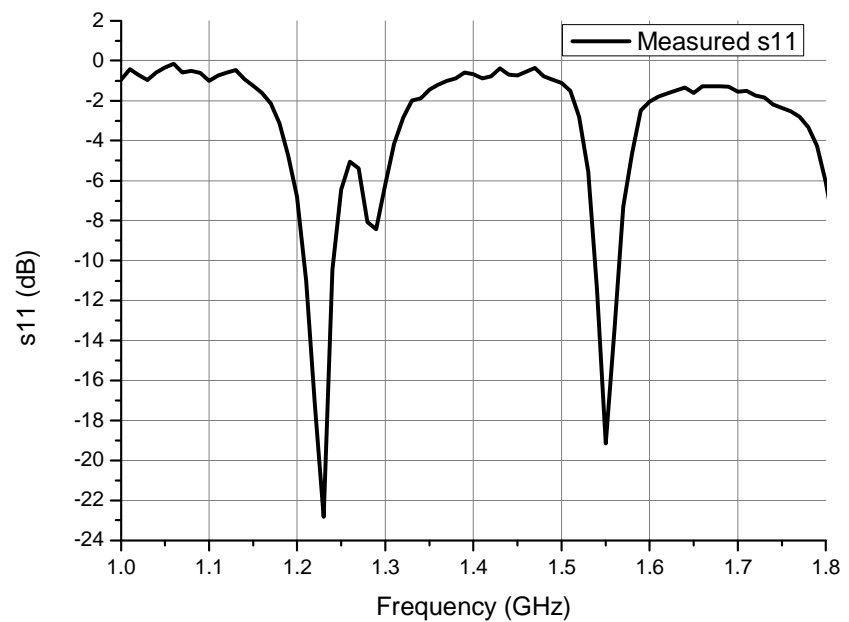

Fig. 10. Measured $s_{11}$ response of S-SAR antenna integrated with CPRGP

It can be seen from Fig. 11 and Fig. 12 that there is a close agreement between simulated and measured results of S-SAR antenna with CPRGP. The discrepancy in the cross polarization around the boresight level can be attributed to the human errors in the antenna manufacturing process. The annular rings had been cut using drills to the close dimensions available. The residual substrate was cut-out using cutting knife which may have deformed the circle into an ellipse thereby disturbing the polarization purity. However, the measured axial ratio at boresight still remains less than $3 \mathrm{~dB}$.

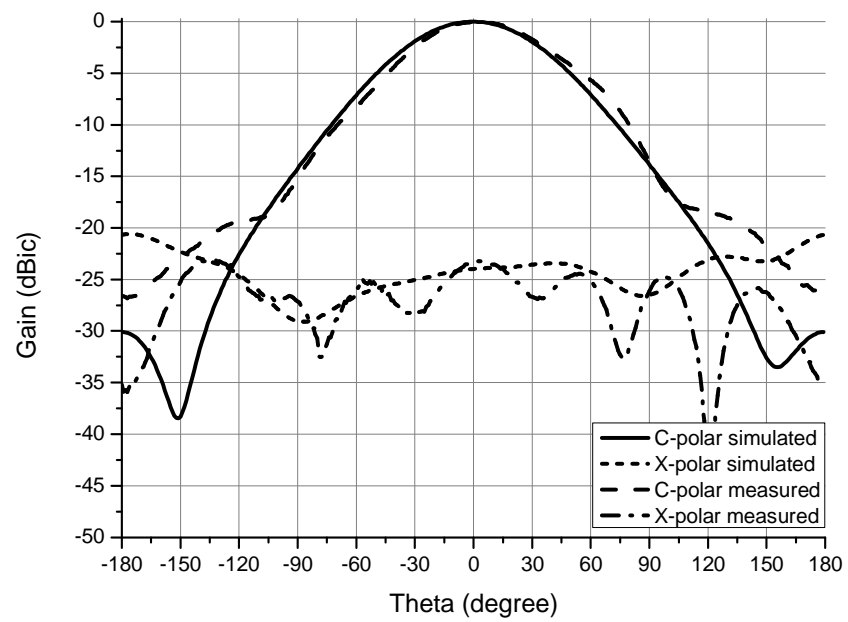

Fig. 11. Comparison of normalized simulated and measured radiation pattern of the S-SAR antenna integrated with CPRGP at $1.227 \mathrm{GHz}$

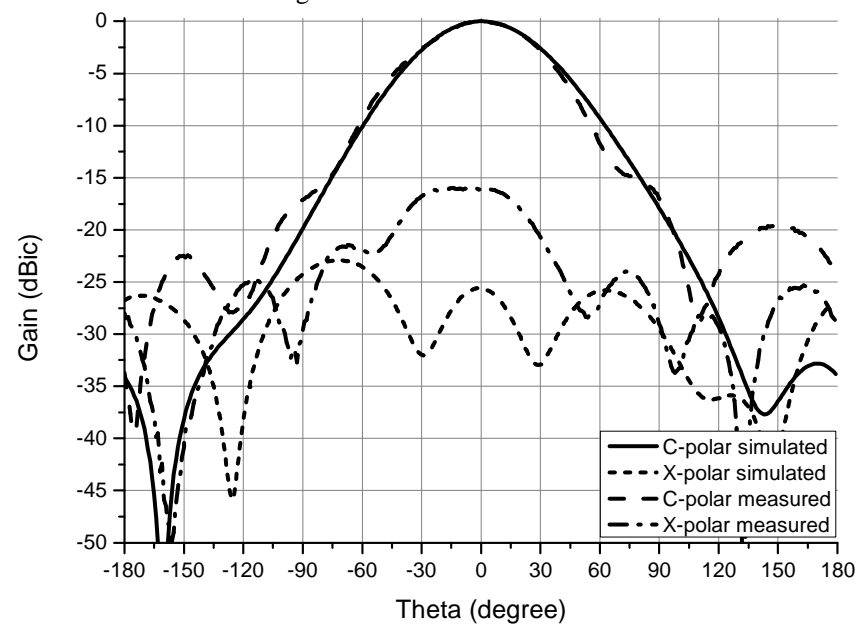

Fig. 12. Comparison of normalized simulated and measured radiation pattern of the S-SAR antenna integrated with CPRGP at $1.575 \mathrm{GHz}$

TABLE I

MEASURED ANTENNA GAIN ACROSS L2 AND L1 BANDS

\begin{tabular}{|c|c|c|}
\hline $\begin{array}{c}\text { Frequency } \\
(\mathrm{GHz})\end{array}$ & $\begin{array}{c}\text { Measured } \\
\text { antenna gain } \\
(\mathrm{dBic})\end{array}$ & $\begin{array}{c}\text { Measured } \\
\text { antenna gain with } \\
\text { CPRGP }(\mathrm{dBic})\end{array}$ \\
\hline 1.217 & 6.0 & 6.1 \\
\hline 1.227 & 5.4 & 5.8 \\
\hline 1.237 & 4.9 & 5.0 \\
\hline 1.565 & 6.7 & 6.8 \\
\hline 1.575 & 7.9 & 7.7 \\
\hline 1.585 & 7.7 & 7.7 \\
\hline
\end{tabular}

\section{MULTIPATH MITIGATION ANALYSIS}

In order to fully appreciate the multipath mitigation capability of the proposed ground plane, a performance comparison of the CPRGP integrated S-SAR antenna against a commercial pinwheel [12] antenna and a non cutoff corrugated ground plane [4] is presented in Table II. Measurement results 
TABLE II

MULTIPATH MITIGATION COMPARISON (ABSOLUTE VALUES)

\begin{tabular}{|c|c|c|c|c|c|c|c|c|c|}
\hline \multirow{2}{*}{\multicolumn{2}{|c|}{ Parameters }} & \multicolumn{2}{|c|}{$\begin{array}{c}\text { Pinwheel Antenna } \\
\text { [12] }\end{array}$} & \multicolumn{2}{|c|}{$\begin{array}{c}\text { Non cutoff } \\
\text { corrugated [4] }\end{array}$} & \multicolumn{2}{|c|}{ S-SAR Antenna } & \multicolumn{2}{|c|}{$\begin{array}{l}\text { S-SAR integrated } \\
\text { CPRGP }\end{array}$} \\
\hline & & L1 & L2 & L1 & L2 & L1 & L2 & L1 & L2 \\
\hline \multicolumn{2}{|c|}{ Antenna Gain (dBic) } & 6.8 & 2.9 & 8 & 8 & 7.9 & 5.4 & 7.7 & 5.8 \\
\hline \multicolumn{2}{|c|}{ FBR $(\mathrm{dB})$} & 32 & 28 & 38 & 38 & 22 & 21.8 & 25 & 27 \\
\hline \multicolumn{2}{|c|}{ Backward radiation (dBic) } & -26 & -25.5 & -30 & -30 & -14.1 & -16.4 & -17.3 & -21.2 \\
\hline \multicolumn{2}{|c|}{ Pattern roll-off $(\mathrm{dB})$} & 13.2 & 11.1 & 18 & 18 & 15 & 11.8 & 18 & 14 \\
\hline \multirow{3}{*}{$\begin{array}{l}\text { Antenna Axial } \\
\text { ratio }(\mathrm{dB})\end{array}$} & $45^{\circ}$ & 1.0 & 2.0 & $\begin{array}{ll}-- \\
\end{array}$ & $\begin{array}{ll}-- \\
-1\end{array}$ & 4.0 & 2.96 & 3.1 & 0.8 \\
\hline & $15^{\circ}$ & 1.4 & 3.8 & --- & --- & 10 & 8.4 & 6.1 & 1 \\
\hline & $5^{\circ}$ & 1.6 & 5.0 & --- & $\begin{array}{ll}-- \\
\end{array}$ & 17 & 12.6 & 7 & 2.5 \\
\hline \multicolumn{2}{|c|}{ RHCP/LHCP @ 90(dB) } & 12 & 8 & 12 & 12 & 5 & 3 & 12 & 11 \\
\hline \multicolumn{2}{|c|}{$\begin{array}{l}\text { Maximum backward cross } \\
\text { polarization }(\mathrm{dBic})\end{array}$} & -9 & -7.5 & -12 & -12 & -7.59 & -14.6 & -18.3 & -17.2 \\
\hline \multirow{2}{*}{$\begin{array}{c}\text { Phase center } \\
\text { variation }(\mathrm{mm})\end{array}$} & Vertical & 0.5 & 1.5 & $<2$ & $<2$ & 0.55 & 1.39 & 0.70 & 0.61 \\
\hline & Horizontal & 0.8 & 1.2 & $<2$ & $<2$ & 2.7 & 0.8 & 1.65 & 0.2 \\
\hline \multicolumn{2}{|c|}{ Ground plane size (mm) } & \multicolumn{2}{|c|}{$200 \times 200 \times 70$} & \multicolumn{2}{|c|}{$340 \times 340 \times 60$} & \multicolumn{2}{|c|}{$150 \times 150 \times 5$} & \multicolumn{2}{|c|}{$190 \times 190 \times 30$} \\
\hline
\end{tabular}

of the standalone S-SAR antenna have also been presented to assess the performance improvement. The non-cutoff (shallow) version of the choke ring ground plane is used in order to appreciate the smaller size. The multipath mitigation performance is compared on the basis of absolute values taken from the CPRGP antenna measurements and the values presented in [4] and [12]. The values for shallow choke ring have been estimated from the measurement plots presented in [4].

Considering the fact that multipath signals consist of both reflected and diffracted signals and can approach the antenna from any arbitrary direction, antenna radiation patterns should make sure that both of these types of signals are significantly attenuated when arriving at the antenna. Let's consider diffracted signals first, the direction of arrival for these signals is near the horizon. The polarization of these signals may primarily be right hand circular and therefore it is vital for the antenna to have low co-polar radiation levels in order to provide maximum attenuation to the incoming signals from this direction. Though for the reflected signals, their polarization state is left hand circular (LHCP) regardless of their direction of arrival and thus it is necessary for the antenna to have sufficiently low cross polarization levels to effectively reject all the incoming reflected signals.

In order to qualify an antenna for multipath interference mitigation the antenna performance is compared with respect to the antenna gain, front to back ratio (FBR), axial ratio, RHCP/LHCP at horizon and maximum level of cross polarization below horizon.

\section{A. Novatel Pinwheel Antenna}

A GPS-704X pinwheel antenna design presented in [12] is a wideband antenna based on an array of coupled spiral slots arranged in a pinwheel configuration. A microstrip multipleturn spiral transmission line arranged on the lower surface of the substrate works as the antenna feed network. The pinwheel antenna is a compact, light weight multipath mitigating antenna with overall dimensions of (including antenna housing) $200 \times 200 \times 70 \mathrm{~mm}^{3}$.

Antenna simulation and measurement results presented in [12] evaluate the pinwheel antenna performance against the antenna gain, pattern roll-off, front-back ratio (FBR), axial ratio and etc. Table II presents the antenna performance in detail and compares it with the proposed CPRGP ground plane. Although the pinwheel antenna is wideband, the performance is compared only for L1 and L2 bands.

\section{B. Non-cutoff corrugated ground plane}

A shallow non-cutoff multipath mitigating antenna presented in [4] combines a wideband droopy bowtie turnstile antenna with a corrugated ground plane to achieve multipath mitigating capability at low elevation angles. The corrugated ground plane is a shallow version of the traditional choke ring ground plane but uses a different approach in order to mitigate multipath interference. Instead of creating high impedance region around the antenna the proposed ground plane allows surface waves to propagate but tunes them so that they are out of phase with the LOS signals at the rim of the antenna and get cancelled. Antenna simulation and measurement results 
presented in [4] investigate the antenna performance against FBR, gain slope, backward radiation and cross polarization isolation. Despite significant reduction in the ground plane thickness, the ground plane has a diameter of $340 \mathrm{~mm}$.

Antenna measurement results are plotted from $1.15 \mathrm{GHz}$ to $1.60 \mathrm{GHz}$ in [4]. Since the measurement results show a similar performance at all frequencies, average values (same) are used in Table II for both L1 and L2 bands.

\section{Performance Comparison}

Multipath mitigation performance of all three antennas is presented in Table II. The performance is compared against the common parameters presented in [4] and [12] which include the front-back ratio, backward radiation, RHCP/LHCP at horizon, cross polarization level below horizon and antenna size. Axial ratio performance of the CPRGP is compared against the pinwheel antenna only as no exact values were found in [4]. It is interesting to note that both FBR and backward radiation are considered as part of the performance comparison, since the front to back ratio of an antenna is defined by both boresight gain and backward radiation. Therefore, a higher FBR may only result from a higher antenna gain and may not reflect better multipath mitigation performance. Since the antenna gain is primarily dependant upon the antenna aperture and may vary for different antenna elements and ground plane sizes, the antenna backward radiation is also included in the performance comparison.

It can be seen from Table II that all three antennae achieve FBR over $25 \mathrm{~dB}$ with backward radiation below $-15 \mathrm{dBic}$.

Antenna axial ratio is another very important factor in determining the antenna's capability to reject multipath interference signals and it is important that a multipath mitigating antenna maintains a good axial ratio performance for the entire upper hemisphere. According to [12] the axial ratio for a multipath mitigating antenna should not exceed; 3 $\mathrm{dB}$ at $45^{\circ}, 6 \mathrm{~dB}$ at $15^{\circ}$ and $8 \mathrm{~dB}$ at $5^{\circ}$ elevation angle. Table II shows that both the pinwheel antenna and the CPRGP achieve required axial ratio performance. The exact values of axial ratio are not available for shallow corrugated ground plane in [4].

Another important factor is the RHCP / LHCP ratio at horizon. This requirement ensures that the antenna is able to receive RHCP signals at the low elevation angles as well. Both shallow corrugated and CPRGP antennae achieve similar performance as presented in Table II.

Antenna cross polarization performance below the horizon is another factor characterizing multipath mitigation performance. This value is important as the majority of reflected (LHCP) signals come from below the horizon and therefore may be accepted by the antenna if sufficient attenuation is not provided. It can be seen that the CPRGP antenna achieves the best performance with maximum cross polar level below the horizon to be $-17 \mathrm{~dB}$.

The last major performance parameter is the antenna phase center variation. The phase center location can vary with both elevation angle and frequency of operation, and its variation is critical for high-precision GNSS applications. A multiband antenna with high variation in the phase center will result in location measurement error as the carrier frequency sweeps through the bandwidth. Similar to [12], the phase center of the CPRGP antenna has been evaluated using the full wave simulation software [11]. Table II shows that maximum variation in the phase center of all three antennas remains less than $2 \mathrm{~mm}$. The presented values for CPRGP are evaluated for the entire upper hemisphere and represent the maximum variation (averaged for $180^{\circ}$ beamwidth) with in the $20 \mathrm{MHz}$ bandwidth at both bands.

Although the CPRGP antenna performance in the front-back ratio and backward radiation may not seem comparable to the shallow corrugated ground plane, it achieves the antenna design requirements presented in [4] and [12]. The performance can be further improved by choosing a more careful manufacturing process.

\section{CONCLUSION}

A new design of a compact multipath mitigating ground plane for multiband GNSS antennae is presented. In order to demonstrate the multipath mitigation performance, the proposed CPRGP is integrated with a dual band step-shorted annular ring antenna. Simulation and measurement results show significant improvement in the antenna co-polar and cross polar performance at both L1 and L2 bands with the ground plane integration. The ground plane performance is also compared against a commercial pinwheel antenna and a shallow corrugated ground plane. Performance comparison presented in Table II show that the CPRGP integrated dualband S-SAR antenna achieves a FBR over $25 \mathrm{~dB}$ with backward radiation below $-17 \mathrm{dBic}$. Cross polar levels below the horizon remain less than $-17 \mathrm{~dB}$. The CPRGP antenna does not replicate the same performance at L1 band due to high directivity and narrow beamwidth at higher frequency. However, the antenna still achieves the required axial ratio performance and therefore qualifies for a good multipath mitigating antenna. Moreover, the ground plane has an advantage in terms of the required space for its accommodation. The overall integrated structure is only $190 \mathrm{x}$ $190 \mathrm{~mm}^{2}$ and weighs only 764 gr. The antenna has a flat bottom surface and is easily mountable on small satellites and platforms. The compact size, low cost and efficient multipath mitigation performance at multiple frequencies makes the proposed CPRGP promising for high precision GNSS applications in small satellites and other small platforms.

\section{REFERENCES}

[1] A. J. Dierendonck, J.P. Fenton and T. Ford, "Theory and performance of narrow correlator spacing in a GPS receiver," ION Journal of Navigation, 39(3), 265-283, 1992.

[2] Basilio, L.I.; Chen, R.L.; Williams, J.T.; Jackson, D.R.; , "A New Planar Dual-Band GPS Antenna Designed for Reduced Susceptibility to LowAngle Multipath," IEEE Transactions on Antennas and Propagation, vol.55, no.8, pp.2358-2366, Aug. 2007 
[3] P. S. Kildal "Artificially soft and hard surfaces in electromagnetics," IEEE Transactions on Antennas and Propagation, vol. 38, no. 10, pp. 1537 - 1544, Oct. 1990

[4] Francesca, Sciré-Scappuzzo and Sergey N. Makarov, “A Low-Multipath Wideband GPS Antenna With Cutoff or Non-Cutoff Corrugated Ground Plane," IEEE Transactions on Antennas and Propagation, vol. 57 No. 1, pp. 33-46, Jan. 2009

[5] M. Maqsood, S. Gao, T. Brown, M. Unwin, "Effects of ground plane on the performance of multipath mitigating antennas for GNSS," Antennas and Propagation Conference (LAPC), 2010 Loughborough, vol., no., pp.241-244, 8-9

[6] Sievenpiper, D. "High-impedance electromagnetic surfaces," Ph.D. Thesis, University of California, Los Angeles, 1999.

[7] R. Baggen, M. Martinez-Vazquez, J. Leiss, S. Holzwarth, L. S. Drioli, P. de Maagt, "Low Profile Galileo Antenna using EBG Technology," IEEE Transactions on Antennas and Propagation, vol. 56, no. 3, pp. 667 - 674, Mar. 2008

[8] D. R. Jackson, J.T. Williams, Arun K. Bhattacharyya, Richard L. Smith, Stephen J. Buchheit and S.A. Long, "Microstrip Patch Designs That Do Not Excite Surface Waves," IEEE Transactions on Antennas and Propagation, vol. 41, pp. 1026-1037, Aug. 1993.

[9] L. Boccia, G. Amendola, G. Di Massa, L. Giulicchi, "Shorted Annular Patch Antenas for Multipath Rejection in GPS-Based Attitude Determination Systems," Microwave and Optical Technology Letters, vol. 28, no. 1, Jan. 2001

[10] Young-Hoon Chun; Jia-Sheng Hong; , "Design of a compact broadband branch-line hybrid," Microwave Symposium Digest, 2005 IEEE MTT-S International, vol., no., pp. 4 pp., 12-17 June 2005

[11] Computer Simulation Technology, Available: www.cst.com

[12] GPS-704X Antenna Design and Performance, Novatel, Available: http://webone.novatel.ca/assets/Documents/Papers/GPS704xWhitePaper.pdf

[13] M. Maqsood, S. Gao, T.Brown, J. D. Xu and J. Z. Li, "Novel Multipath Mitigating Ground Planes for Multi-band Global Navigation Satellite System Antennas," Proc. of European Conf. on Antennas and Propagation, Mar. 2012 